\title{
Dopamine Transporter Gene Associated with Diminished Subjective Response to Amphetamine
}

\author{
David C Lott ${ }^{1,2}$, Soo-Jeong Kim', Edwin H Cook Jr ${ }^{1,3}$ and Harriet de Wit*,1 \\ 'Department of Psychiatry, The University of Chicago, Chicago, IL, USA; ${ }^{2}$ Department of Psychiatry, The University of Illinois at Chicago, IL, USA; \\ ${ }^{3}$ Departments of Pediatrics and Human Genetics, The University of Chicago, IL, USA
}

\begin{abstract}
Individual variability in responses to stimulant drugs may influence risk of stimulant abuse and treatment response. However, the genetic determinants of this variability have yet to be elucidated. The dopamine transporter is an important site of amphetamine action. Therefore, the dopamine transporter gene (DATI) is a logical candidate gene to study. Using a drug challenge approach, we tested for association between DATI genotype and subjective responses to amphetamine in healthy adults. Volunteers participated in a doubleblind, crossover design, randomly receiving placebo, $10 \mathrm{mg}$, and $20 \mathrm{mg}$ oral D-amphetamine, and completed self-report measures on subjective effects including anxiety and euphoria. Subjects were genotyped for the DATI 3'-untranslated region VNTR polymorphism and divided into groups based on genotype: homozygous for nine repeats $(9 / 9, N=8)$, heterozygous $(9 / 10, N=36)$ and homozygous for 10 repeats $(10 / 10, N=52)$. The effects of amphetamine on ratings of Feel Drug, Anxiety, and Euphoria were examined with ANCOVA. In 9/10 and 10/10 subjects, amphetamine produced its expected effects of increased Euphoria, Anxiety, and Feel Drug $(p<0.0 \mathrm{I})$. However, in $9 / 9$ subjects, the effects of amphetamine were indistinguishable from placebo, suggesting that the 9/9 genotype has diminished subjective response to acute amphetamine. Interestingly, recent findings also implicate the 9/9 genotype in decreased therapeutic response to the stimulant methylphenidate in ADHD children. The current findings have important implications for understanding the genetic determinants of variability in stimulant response and risk of abuse.

Neuropsychopharmacology (2005) 30, 602-609, advance online publication, 15 December 2004; doi: I 0.1038/sj.npp. 1300637
\end{abstract}

Keywords: dopamine transporter; amphetamine; genetic; substance abuse; stimulant; pharmacogenetic

\section{INTRODUCTION}

Individuals vary markedly in their subjective and behavioral responses to stimulant drugs. Variability has been reported in clinical settings, such as in response to treatment for Attention-Deficit Hyperactivity Disorder (Spencer et al, 1996) and narcolepsy (Mitler et al, 1993). Individual variability has also been reported in the mood-enhancing effects of stimulants, which are thought to be linked to their potential for abuse (Brauer and de Wit, 1996; de Wit et al, 1986; Gabbay, 2003). There are many potential sources of this variability, including expectancies (Mitchell et al, 1996), personality (Hutchison et al, 1999), psychiatric symptomatology (Cesarec and Nyman, 1985), sex, and menstrual cycle phase (White et al, 2002). One further factor that is receiving considerable attention recently is the possibility

\footnotetext{
*Correspondence: Dr $\mathrm{H}$ de Wit, Department of Psychiatry, The University of Chicago, 584I S. Maryland Avenue MC3077, Chicago, IL 60637, USA, Tel: + I 773702 I537|; Fax: + I 773834 7698; E-mail: hdew@uchicago.edu

Received 21 June 2004; revised 7 October 2004; accepted 21 October 2004

Online publication: 8 November 2004 at http://www.acnp.org/citations/ Npp I 10804040285/default.pdf
}

that people vary in responses to drug because of genetic variations in the function of proteins involved in neurotransmitter action (eg Mattay et al, 2003).

A likely source of genetic variation influencing the effects of amphetamine is in the dopamine system, where amphetamine acts by altering transporter function, blocking reuptake, and causing reverse transport of intracellular dopamine, as well as by inducing vesicular release of dopamine (Jones et al, 1998). Several lines of evidence link variations in responses to amphetamine to function of the dopamine transporter. For example, knockout mice lacking the dopamine transporter show a reduced locomotor response to amphetamine (Spielewoy et al, 2001). In humans, polymorphisms in the dopamine transporter gene (DAT1) are associated with variations in both therapeutic and adverse responses to other stimulants, such as cocaine and methylphenidate (Gelernter et al, 1994; Kirley et al, 2003; Stein et al, 2002).

DAT1 has a VNTR polymorphism in the $3^{\prime}$-untranslated region (3'-UTR) (Vandenbergh et al, 1992) that has been shown to affect gene expression. Although there have been some conflicting reports (Jacobsen et al, 2000; Martinez et al, 2001), the longer 10-repeat allele appears to cause greater DAT1 expression than the nine-repeat allele (Fuke 
et al, 2001; Heinz et al, 2000; Mill et al, 2002). Further, the one study examining the nine-repeat homozygous genotype separately found that this group had lower expression than either 10-repeat homozygotes or 9/10 heterozygotes (Mill et al, 2002).

Several studies have examined the relationship of the $3^{\prime}$-UTR VNTR polymorphism with response to stimulants. Gelernter et al (1994) reported that cocaine users with the $9 / 9$ and $9 / 10$ genotypes were more likely to experience cocaine-induced paranoia than cocaine users with the 10/10 genotype. In most studies of response to methylphenidate in ADHD youth (Kirley et al, 2003; Loo et al, 2003; Stein et al, 2002), the clinical response was poorer in patients with the $9 / 9$ and $9 / 10$ genotypes compared to the 10/10 genotype, although two studies reported increased therapeutic response in the presence of nine-repeat alleles (Roman et al, 2004; Winsberg and Comings, 1999). From these studies it is not yet clear whether the nine-repeat or the 10-repeat allele is dominant. Notably, whereas most studies have grouped individuals with any nine-repeat alleles together (ie 9/9 and 9/10) (Kirley et al, 2003; Loo et al, 2003; Roman et al, 2004; Winsberg and Comings, 1999), Stein et al (2002) analyzed the nine-repeat homozygotes separately, and found that this group of ADHD patients exhibited a substantially dampened response to increasing doses of methylphenidate treatment, compared with either the $9 / 10$ or $10 / 10$ patients. This calls into question the previous combining of $9 / 9$ and $9 / 10$ to match the sample size of the $10 / 10$ sample. Differing results among studies could be due to different proportions of $9 / 9$ and $9 / 10$ when the two are combined.

In the present study, we examined the interaction between amphetamine and DAT1 3'-UTR genotype using single doses of D-amphetamine in healthy volunteers, under highly controlled laboratory conditions. Extraneous factors such as expectancies, variations in subject characteristics, environmental influences, and drug dosing were controlled, and sensitive instruments were used to detect effects on a range of outcome measures. Based on the findings reviewed above, we hypothesized that the $9 / 9$ genotype at DAT1 $3^{\prime}$ UTR would differ from other groups in their subjective response to acute doses of amphetamine, but the direction of the difference in response was not easy to predict.

\section{MATERIALS AND METHODS}

\section{Subjects}

Healthy male and female volunteers $(N=101)$, aged 18-35 years, were recruited by posters, advertisements, and wordof-mouth referrals. To reduce variability due to tolerance or withdrawal from nicotine or caffeine, volunteers were excluded if they smoked more than 10 cigarettes per week or consumed more than three cups of coffee per day. Candidates underwent a structured clinical psychiatric interview and a physical examination, and completed several screening questionnaires. They also completed a psychiatric symptom checklist (SCL-90; (Derogatis, 1983)), the Michigan Alcoholism Screening Test (Selzer, 1971), and a health questionnaire with a detailed section on current and lifetime drug use. All subjects obtained an electrocardiogram and received a physical examination by a physician. Volunteers were excluded if they had any current medical condition requiring medication, any current or past medical condition considered to be a contraindication for amphetamine (eg hypertension, abnormal EKG), any current Axis I psychiatric disorder (American Psychiatric Association, 1994, DSM IV), if they had been treated for a substance use disorder or had a history of legal, personal or employment problems related to drug use, if they had less than a high school education, were not fluent in English, or if they worked a night shift. Women who were pregnant or lactating, or planning to become pregnant during the study were excluded.

\section{Design}

The study used a three-session crossover design, in which each subject received placebo and D-amphetamine (10 and $20 \mathrm{mg}$ ), under double-blind conditions and in randomized order. The subjective, physiological, and behavioral effects of amphetamine were recorded over $4 \mathrm{~h}$ after drug administration. All subjects were genotyped after all subjects had completed the behavioral phase of the study. Genotyping was performed blind to all behavioral data.

\section{Procedure}

Subjects first attended an orientation session to provide informed consent, practice tests, and questionnaires, complete a personality questionnaire, and give a blood sample for genotyping. Subjects were instructed to abstain from taking drugs, including alcohol, nicotine, and caffeine, for $24 \mathrm{~h}$ before each session and to fast from midnight the night before the sessions. Subjects were tested individually, and remained in a comfortably furnished room with television and reading materials for the 4-h session. Subjective and behavioral tasks were administered via computer. Volunteers could watch emotionally neutral movies and read during the sessions when measurements were not being taken. The study was approved by the Institutional Review Board of The University of Chicago and was carried out in accordance with the Helsinki Declaration of 1975.

The three sessions were conducted from 0900 to 1300 , at least $48 \mathrm{~h}$ apart. Women were tested during the follicular phase of their menstrual cycle because responses to amphetamine are dampened during the luteal phase (White et al, 2002). Upon arrival in the laboratory for each session, subjects provided urine and breath samples to confirm drug and alcohol abstinence, and women were tested for pregnancy. Subjects were given a light meal (bagel with cream cheese) to reduce stomach irritation from the drug. They completed precapsule baseline measures including subjective, behavioral, and physiologic measures. At 0930, subjects ingested a capsule containing D-amphetamine (10 or $20 \mathrm{mg}$ ) or placebo with $100 \mathrm{ml}$ of water. At $30,60,90,150$, and $180 \mathrm{~min}$ after capsule ingestion, heart rate, blood pressure, and temperature were measured, and subjects completed ratings of drug effects and mood (see below). At 90 min after capsule ingestion, subjects completed two behavioral tasks measuring impulsive behavior, which will be reported elsewhere. At 1300, subjects left the laboratory. Previous studies in this laboratory indicate that the subjective and physiological effects of D-amphetamine 
return to near baseline levels within $4 \mathrm{~h}$ of oral administration. After completing the three sessions, subjects were debriefed and paid.

\section{Dependent Measures}

Subjective drug effects were assessed using three standardized instruments - the Drug Effects Questionnaire (DEQ), the Profile of Mood States (POMS; Johanson and Uhlenhuth, 1980; McNair et al, 1971), and the 49-item Addiction Research Center Inventory (ARCI; Martin et al, 1971). The DEQ consists of four Visual Analog Scales (VAS) on which subjects rated whether they feel the drug, like the drug, feel high, and want more. Each scale consisted of a $100 \mathrm{~mm}$ line labeled 'no drug effect at all' at one end and 'strong effect' at the other (Johanson and Uhlenhuth, 1980). The POMS (Johanson and Uhlenhuth, 1980; McNair et al, 1971) is a 72item adjective checklist with a scale sensitive to several mood states, including anxiety, depression, vigor, fatigue, friendliness, anger, elation, arousal, confusion, and positive mood. The ARCI (Martin et al, 1971) consists of true-false questions designed to measure drug-specific effects on five scales: the amphetamine (A) scale, which assesses effects of amphetamine; the benzedrine group (BG) scale, an amphetamine-sensitive scale measuring intellectual efficiency and energy; the morphine-benzedrine group (MBG) scale, a measure of euphoria; the pentobarbital-chlorpromazinealcohol group (PCAG) scale, which tests levels of sedation, and the lysergic acid diethylamide (LSD) scale, a measure of dysphoria and somatic complaints (Foltin and Fischman, 1991). From these questionnaires, the three primary measures were selected to capture the prototypic effects of amphetamine: the DEQ Feel Drug scale, the POMS Anxiety scale, and the ARCI MBG (Euphoria) scale.

Physiological effects, including heart rate and blood pressure, were measured before capsule administration and at repeated intervals in the $4 \mathrm{~h}$ after the capsule as described above.

\section{Genotyping}

PCR was carried out in a $10 \mu \mathrm{l}$ volume containing $50 \mathrm{ng}$ of genomic DNA, $0.5 \mu \mathrm{M}$ of each primer, one of which was $5^{\prime}$ fluorescently labeled, $200 \mu \mathrm{M}$ of each dNTPs, $1 \times$ PCR buffer, $1.5 \mathrm{mM} \mathrm{MgCl}_{2}, 0.3 \mathrm{U}$ of DyNAzyme ${ }^{\mathrm{TM}}$ EXT DNA polymerase (Finnzymes Oy, Espoo, Finland) and 0.5 M GCmelt (Clontech, Palo Alto, CA, USA). Primer sequences were $5^{\prime}$-TGTGGTGTAGGGAACGGCCTGAG- $3^{\prime}$ (sense) and $5^{\prime}$ CTTCCTGGAGGTCACGGCTCAAGG- $3^{\prime}$ (antisense). PCR cycle included an initial denaturation at $96^{\circ} \mathrm{C}$ for $12 \mathrm{~min}$, followed by 45 cycles consisting of $96^{\circ} \mathrm{C}$ for $30 \mathrm{~s}, 68^{\circ} \mathrm{C}$ for $45 \mathrm{~s}$, and $72^{\circ} \mathrm{C}$ for $3 \mathrm{~min}$, with a final extension at $72^{\circ} \mathrm{C}$ for 10 min. PCR products were separated on an ABI PRISM 3700 Genetic Analyzer at the University of Chicago DNA Sequencing and Genotyping Core. The genotypes were determined using Genotyper software (version $3.7 \mathrm{NT}$ ) (Applied Biosystems, Foster City, CA, USA).

\section{Data Analysis}

Subjects were genotyped at the $3^{\prime}$-UTR VNTR polymorphism of DAT1 and were categorized into three groups (9/9,
9/10, and 10/10). Of 101 study participants, one was excluded because of a missing laboratory sample, and four others because of having rare alleles. Subjective measures were compared for the three genotyped groups using separate repeated-measure ANCOVAs (SPSS 11.0) for each of the outcome measures. Genotype was the grouping factor, baseline (predrug) scores were used as covariates, and time (five time points after capsule ingestion) and dose $(0,10$, and $20 \mathrm{mg})$ were the two within-subjects factors. In addition, separate one-way ANOVAs were performed using AUC for all 96 subjects together to assess for the presence of drug response on the primary outcomes when a drug main effect was not apparent. This additional ANOVA was necessary in light of the small $9 / 9$ group $(n=8)$, which limited the power of the initial analyses. All post hoc analyses were conducted with independent samples $t$-test (SPSS 11.0 and Graphpad Quickcalcs) or paired samples $t$ tests as appropriate (SPSS 11.0). Alpha was set at $p<0.05$ (two-tailed) for all analyses.

Demographic characteristics for the three groups were compared using ANOVA or $\chi^{2}$ tests. For measures on which the groups differed, Pearson's $r$ correlations were conducted for continuous data (current alcohol use) and independent samples $t$-tests for dichotomous data (current marijuana use), with difference in AUC between placebo and each drug dose as the dependent variables to determine whether these differences contributed to the observed group differences in outcome measures. In addition, baseline differences across genotype groups on the three primary outcome measures were assessed using one-way ANOVA, with placebo trial predrug score as the dependent measure.

Hardy-Weinberg equilibrium was analyzed using on-line resources (http://www.kursus.kvl.dk/shares/vetgen/_Popgen/ genetik/applets/kitest.htm).

\section{RESULTS}

\section{Subjects}

Allele and genotype frequencies for nine- and 10- repeat alleles are shown in Table 1. Four subjects were excluded from the analyses because they had rare alleles (one with seven-repeat allele; two with eight-repeat allele; one with 11-repeat allele, all heterozygous with a 10-repeat allele). The blood sample for one subject was lost. One subject in the 9/9 group was excluded from analysis on Euphoria because responses were consistently at the top of the scale, indicating invalid reporting on this scale. The three genotypes represented by the two most common alleles included in the analyses did not significantly deviate from

Table I Allele and Genotype Frequencies for DATI VNTR Polymorphism for 96 Subjects with Common Genotypes

\begin{tabular}{lcccc}
\hline \multicolumn{2}{c}{ Allele } & \multicolumn{3}{c}{ Genotype } \\
\hline $9 \mathrm{R}$ & $\mathrm{IOR}$ & $9 / 9$ & $9 / 10$ & $10 / 10$ \\
$52(27.1 \%)$ & $140(72.9 \%)$ & $8(8.3 \%)$ & $36(37.5 \%)$ & $52(54.2 \%)$
\end{tabular}

Four additional subjects had one rare allele (one with seven-repeat allele; two with eight-repeat allele; one with II-repeat allele, all heterozygous with 10-repeat allele). Frequencies are similar to those reported previously. 
Table 2 Demographics Characteristics and Drug Use for Subjects in the Three Genotype Groups, 9/9, 9/10, and 10/10

\begin{tabular}{|c|c|c|c|}
\hline $\begin{array}{l}\text { Demographic } \\
\text { characteristics }\end{array}$ & Group $9 / 9$ & Group $9 / 10$ & Group $10 / 10$ \\
\hline$n$ & 8 & 36 & 52 \\
\hline Age (mean years) & $26.9 \pm 2.4$ & $23.4 \pm 0.5$ & $23.7 \pm 0.6$ \\
\hline \multicolumn{4}{|l|}{ Ethnicity } \\
\hline$\%$ White & 62 & 75 & 50 \\
\hline$\%$ Black & 25 & 11 & 21 \\
\hline$\%$ Asian & 0 & 3 & 19* \\
\hline \% Latino & 12 & 8 & 15 \\
\hline Gender (\% male) & 62 & 53 & 46 \\
\hline Education (mean years) & $14.5 \pm 0.5$ & $15.6 \pm 0.3$ & $14.8 \pm 0.2$ \\
\hline \multicolumn{4}{|l|}{ Current substance use } \\
\hline Alcohol (mean dr/wk) & $3.8 \pm 1.1$ & $5.3 \pm 0.6 *$ & $3.5 \pm 0.4$ \\
\hline Cigarettes (mean cig/wk) & $0.8 \pm 0.4$ & $1.3 \pm 0.5$ & $0.8 \pm 0.3$ \\
\hline Caffeine (mean cups/d) & $2.0 \pm 0.4$ & $1.3 \pm 0.2$ & $1.3 \pm 0.2$ \\
\hline Marijuana (\% yes) & 12 & $42 *$ & 15 \\
\hline \multicolumn{4}{|l|}{ Lifetime substance use } \\
\hline Stimulants (\% ever used) & 0 & 19 & 19 \\
\hline Sedatives (\% ever used) & 0 & 11 & 4 \\
\hline Opiates (\% ever used) & 12 & 17 & 12 \\
\hline Marijuana (\% ever used) & 87 & 78 & 69 \\
\hline
\end{tabular}

Comparisons across the groups were made using one-way ANOVA for continuous data and $\chi^{2}$ tests for frequency data $\left({ }^{*} p<0.05\right)$.

Hardy-Weinberg Equilibrium ( $\left.1 \mathrm{df}, \chi^{2}=0.2453, p>0.2\right)$. Table 2 shows the demographic characteristics of the 96 subjects included, grouped by genotype.

The three groups were similar on most demographic measures including age, gender, and education. However, consistent with Kang et al (1999), none of the subjects in the 9/9 group were Asian, whereas 2.8 and $19.2 \%$ of the $9 / 10$ and $10 / 10$ groups, respectively, were Asian $(p<0.05)$. The groups reported similar current drug use, except that the $9 /$ 10 group reported significantly higher current use of alcohol and marijuana $(p<0.05)$. In separate analyses, we found no relationship between current alcohol or marijuana use and responses to amphetamine on each of the three primary outcome measures. Baseline (ie precapsule) scores did not differ across the genotype groups.

\section{Overall Amphetamine Effects}

When data from all subjects were considered together, amphetamine produced its expected effects on all three primary outcome measures, with increased Euphoria and Feel Drug $(p<0.001$, drug main effect from repeatedmeasures ANCOVA), and increased Anxiety $(p<0.02$, oneway ANOVA with AUC). Table 3 summarizes the measures on which significant drug or group effects were obtained, and shows that amphetamine significantly increased scores on ARCI A, BG, and MBG scales; POMS Arousal, Elation, Friendliness, Positive Mood, and Vigor; and DEQ Feel Drug, High, and Want More; and decreased scores on sedation (ARCI PCAG scale) in the ANCOVA analysis.

For most measures, the drug effects appeared by $60 \mathrm{~min}$ and peaked between 90 and $120 \mathrm{~min}$ (see Figure 2). On most measures the effects were also dose-dependent.

\section{Genotype Differences in Drug Effects}

The effects of D-amphetamine on the three primary outcome measures varied across genotype groups, although differences did not reach statistical significance on all measures (Table 3, Figure 1). Amphetamine increased ratings of Feel Drug (DEQ) in the $9 / 10$ and $10 / 10$ groups but not in the $9 / 9$ group. On this measure, the group $\times$ drug effect was significant at $p<0.02$, and post hoc comparisons revealed significant drug response compared to placebo at both doses for the 9/10 and 10/10 groups but not the 9/9 group $(p<0.05$ for low dose and $p<0.0005$ for high dose for both the $9 / 10$ and $10 / 10$ groups).

The results also suggest a similar pattern for Anxiety and Euphoria. The group $\times$ drug interaction for Anxiety trended toward significance $(p<0.09)$, but no post hoc comparisons were made because of failure to reach the 0.05 significance level. However, visual inspection of the graph reveals a pattern like that for Feel Drug, with diminished response in the $9 / 9$ group (Figure 1).

In a similar manner, the group $\times$ drug interaction for Euphoria did not reach statistical significance, although there was a significant drug main effect $(p<0.005)$. Again, the graph reflects a similar pattern of less response in the $9 / 9$ group than in the $9 / 10$ and $10 / 10$ groups (Figure 2). For Euphoria, one subject in the 9/9 group was excluded from analyses because the responses were consistently at the maximum of the scale throughout testing, indicating invalid responding. Owing to the already low sample size for the 9/9 group, this exclusion further reduced power to detect differences. Analysis of Euphoria with this subject included produced marginally lower $p$-values.

Analysis of secondary subjective measures revealed two significant group effects or interactions involving group (Table 3 ). First, the ARCI LSD scale had a group $\times$ drug effect $(p<0.015)$. Post hoc analyses demonstrated that amphetamine $(20 \mathrm{mg})$ increased LSD scores in the $10 / 10$ group $(p<0.0005)$ but not the $9 / 10$ or $9 / 9$ groups (NS).

Genotype groups also differed on the DEQ measure High. Although there were no interactions with group and drug, visual inspection of the data and post hoc tests suggested that amphetamine $(20 \mathrm{mg})$ produced higher scores in the $10 / 10$ group than this dose in the $9 / 10$ group $(p<0.05$ for group effect and post hoc), while the groups did not differ in scores at amphetamine $(10 \mathrm{mg})$ or placebo. The responses of the $9 / 9$ group were intermediate between the $9 / 10$ and $10 / 10$ groups.

In the analysis of physiological measures, amphetamine differentially increased diastolic blood pressure (DBP) with a group $\times$ drug effect of $p<0.005$. As with the primary outcomes Feel Drug and Anxiety, the effects of drug on DBP were nonsignificant in the $9 / 9$ group in contrast to the other groups based on post hoc analyses $(p<0.01$ for high-dose 
Table 3 F-Values for Scales on which Significant Drug or Group Effects were Obtained

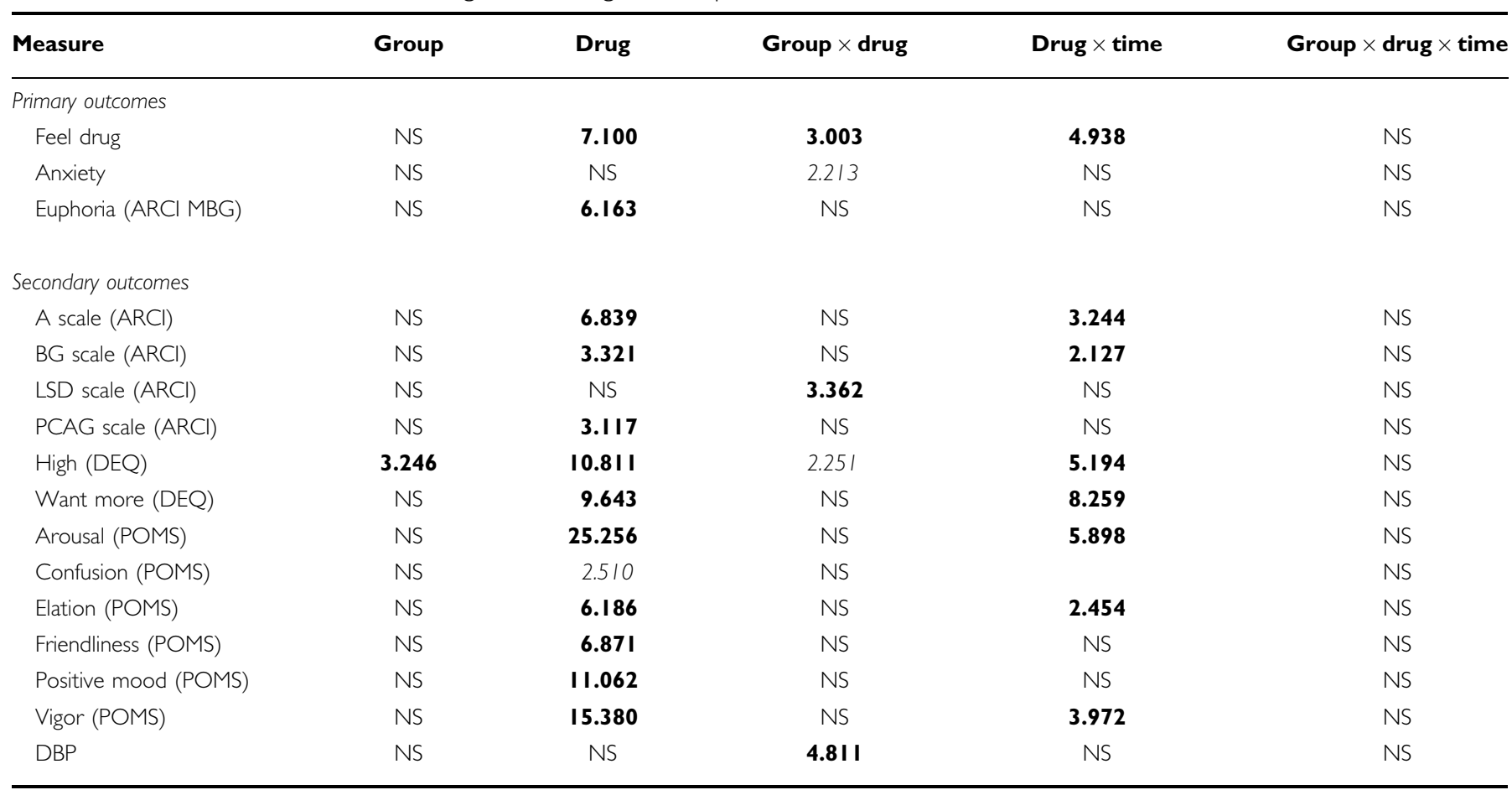

Bold numbers refer to significant effects $(p<0.05)$; italics refer to trends (ie $p<0.10$ ); NS = not significant; DBP = diastolic blood pressure.

drug response). There were no significant results for systolic blood pressure or heart rate.

When ANCOVA analyses of primary outcomes were repeated with all Asian subjects excluded, the results were unchanged.

\section{DISCUSSION}

The primary finding of this study was that healthy volunteers homozygous for the nine-repeat allele of DAT1 exhibited a pattern of diminished responsiveness to the subjective effects of acute amphetamine. This was especially apparent on measures of global drug effect (Feel Drug), feeling High, and a measure of dysphoria (ARCI LSD), and it was also evident to a lesser extent on measures of Anxiety (POMS; Figure 1) and Euphoria (ARCI MBG; Figure 2). Subjects homozygous for the nine-repeat allele also exhibited a diminished drug response on a physiological measure, DBP. Some differences detected may have resulted from greater response of $10 / 10$ subjects, but post hoc comparisons and overall review of the results support the presence of a particularly lower response in the 9/9 group.

Apart from the apparent differences in responses to amphetamine, the three genotyped groups also differed in certain measures independent of drug administration. In particular, the $9 / 10$ group reported significantly higher current alcohol and marijuana use than either of the other two groups (Table $1, p<0.05$ ), and the proportion of Asian subjects varied across the groups. There are several reasons to believe that the differences in alcohol and marijuana use were probably due to chance, and not related to our primary findings. First, the higher drug use occurred in the $9 / 10$ group, whereas the differential responses to amphetamine occurred in the 9/9 group. Second, separate correlational analysis showed that habitual use of alcohol and marijuana was not related to responses to amphetamine, in the entire sample. Third, it is unusual for a strong genetic influence to occur in only the heterozygous group. These considerations make it unlikely that the differences in drug use contributed to our findings. The differences in proportions of Asians in the three genotyped groups has been reported previously (Kang et al, 1999). A secondary analysis of primary outcomes excluding all Asians yielded identical results, demonstrating that this ethnic variation did not influence our findings.

If DAT1 genotype plays an important role in acute responses to stimulant drugs, one may ask why the group differences were not apparent on more of the outcome measures. The genotype-behavior relationship may be modest for several reasons. First, amphetamine acts on several other neurotransmitter systems in addition to its dopaminergic actions. Thus, genetic variation in a single receptor system is likely to account for only some of the individual variability in its effects. Second, the primary outcome measures in the present study were self-reports of mood and subjective states such as anxiety and feeling high, which are typically associated with relatively high variability, both within and between subjects. Therefore, the magnitude of an effect of genotype must be large to overcome this variability. Third, the small number of subjects $(N=8)$ in the $9 / 9$ group greatly diminished the power to detect group differences, a problem made worse for Euphoria because of the exclusion of one 9/9 subject on this measure. While the confounding effect of amphetamine's multiple sites of action, the inherent variability of 

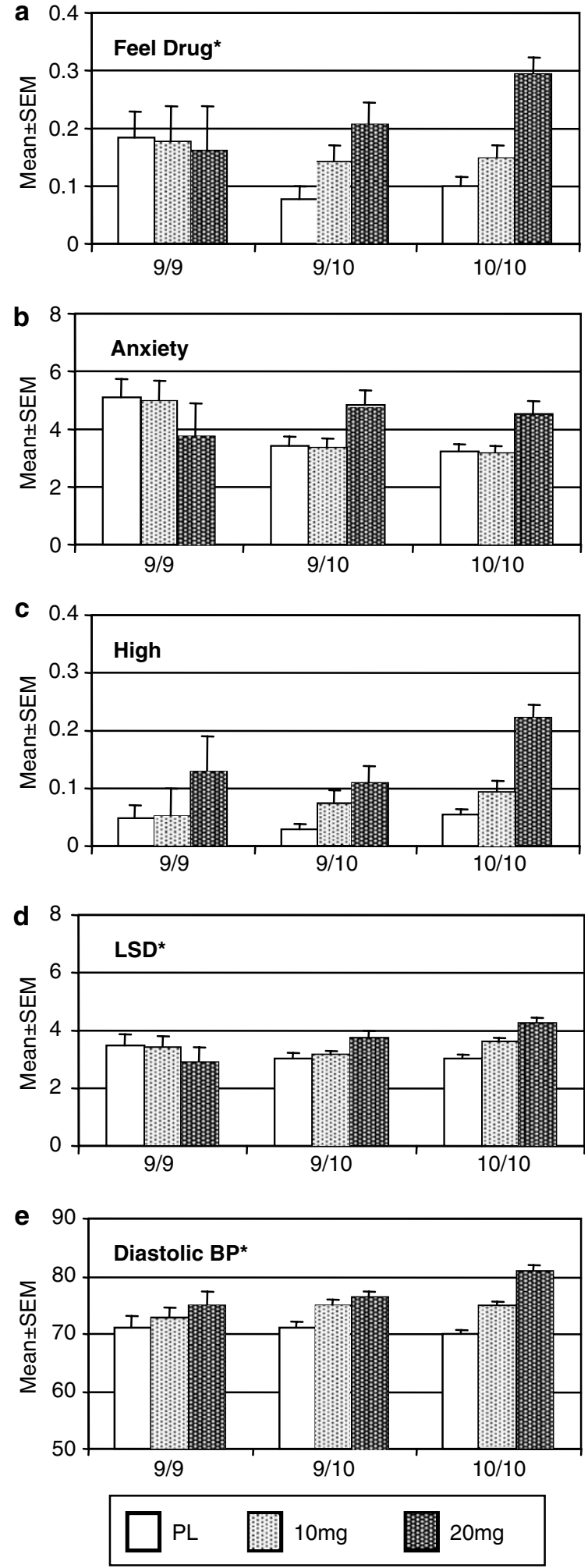

Figure I Mean \pm SEM scores on selected measures of drug effects, after placebo, $10 \mathrm{mg}$, and $20 \mathrm{mg}$ D-amphetamine in healthy volunteers, grouped by DATI genotypes ( $x$-axis). The number of subjects in each group was $9 / 9(n=8), 9 / 10(n=36)$, and 10/10 $(n=52)$. The drug significantly increased overall mean scores on each of these measures. Visual inspection of the means indicates that responses to $D$-amphetamine were attenuated in the 9/9 group. *Group $\times$ drug effect, $p<0.05$.

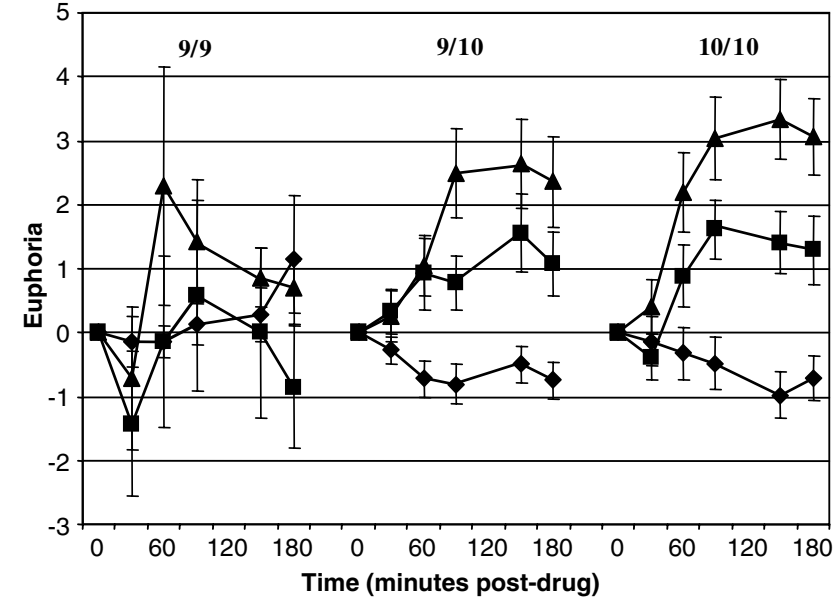

Figure 2 Mean $( \pm S E M)$ ratings on the MBG scale of the $A R C l$, a measure of Euphoria, in the three genotype groups after placebo $(\bullet)$, $10 \mathrm{mg}(\boldsymbol{\square})$, and $20 \mathrm{mg}(\boldsymbol{\Lambda})$ amphetamine. Mean ratings are shown as change in ratings from before drug administration (indicated as time 0), through $180 \mathrm{~min}$ after ingestion of the capsule. The drug significantly increased ratings on this measure (drug effect, $p<0.005$ ).

subjective reports, and the small 9/9 sample size all limited the power of our study, several significant genotype group differences were found.

One compelling reason to believe that these results reflect a true difference in drug effects between the groups is the consistency of these findings with studies of methylphenidate in ADHD patients. Stein et al (2002) observed a markedly diminished response to methylphenidate treatment in a prospective study of ADHD children. While methylphenidate dose-dependently reduced parental symptom ratings in $9 / 10$ and $10 / 10$ children, children with two nine-repeat alleles had little therapeutic response. Furthermore, two studies have reported lower response to methylphenidate in ADHD patients with the 9/9 genotypes combined with the $9 / 10$ genotypes using EEG measures (Loo et al, 2003) and parental report clinical measures (Kirley et al, 2003). The finding here of a similar pattern using amphetamine supports the previous findings of diminished response to stimulant action in nine-repeat homozygotes. The dopamine transporter is an important part of the therapeutic action of both methylphenidate and amphetamine, and homozygosity for the $3^{\prime}$-UTR polymorphism nine-repeat allele may predict therapeutic nonresponse for both stimulants. Although two other studies have reported increased therapeutic response in the presence of any nine-repeat alleles (Roman et al, 2004; Winsberg and Comings, 1999), studies such as these which grouped the 9/9 genotypes together with the $9 / 10$ genotypes are difficult to interpret. Variation in previous studies may have been due to mixing of the $9 / 9$ and $9 / 10$ genotypes, and future studies should avoid assumptions about the dominance of the nine- or 10-repeat allele.

The present findings may have implications for individual differences in susceptibility to substance abuse. There is evidence that individual differences in acute drug responses (eg pleasurable $v s$ nonpleasurable effects) are predictive of drug use. For example, initially positive responses to opiates or cannabis on first use have been linked to susceptibility to 
use (Fergusson et al, 2003; Haertzen et al, 1983). Although it has been difficult to detect associations between DAT1 and substance abuse or dependence (Uhl et al, 2002), this may be because the phenotype of drug abuse is multifactorial, and many other nonbiological and nongenetic factors contribute to the development of drug use. Instead, the endophenotype of acute drug response may be a more sensitive measure of genetic determinants of abuse and dependence. In the case of DAT1 and amphetamine, this study supports a view that homozygosity for the nine-repeat allele would likely confer protection from dependence on amphetamine because of a lack of reactivity to the drug.

In this study, we have used a method combining acute pharmacological challenge and genetic association to study the genetic determinants of acute drug response. In addition to the study of risk of addiction, this experimental method may also be valuable for predicting therapeutic responses to drugs, particularly when the drug has therapeutic value in acute administration, as with stimulants. A similar approach has been taken recently by Mattay et al (2003), who combined this drug challenge method with brain imaging to show that the cognitive effects of an acute dose of amphetamine were associated with catechol $O$-methyltransferase genotype. This method may also be valuable for the study of disease risk whenever an acute drug response is an endophenotype for a disease, as in the case of addiction.

Our results have important implications for future analyses of the DAT1 3'-UTR VNTR. Most prior studies have grouped $9 / 9$ and $9 / 10$ subjects together because of the low occurrence of the 9/9 genotype. However, our results show the importance of analyzing $9 / 9$ subjects separately. This is particularly true since our results suggest that the $9 / 10$ group respond more like the $10 / 10$ group than the $9 / 9$ group. Thus, combining the $9 / 9$ and $9 / 10$ groups may mask important differences unique to the $9 / 9$ group. In other words, the 10-repeat allele appears to behave as a dominant allele with respect to acute stimulant response as supported by the results reported here and by Stein et al (2002). Paradoxically, the lack of a positive mood response to stimulants may confer protection from stimulant abuse, while increasing the risk of nonresponse to stimulant treatment of ADHD. This is consistent with the idea that risk alleles for one phenotype (ie nonresponse to stimulant treatment) may confer advantages in other contexts (ie decreased liability to stimulant abuse).

It is important to consider several limitations of this study. First, the main limitation of this study is the small number in the 9/9 group, which limited the power to detect significant group effects and interactions and lowered the power of finding other effects in the ANCOVA analyses. In future studies, it may be preferable to identify sufficient numbers of subjects with rare genotypes. Second, the study is limited by the selected population of subjects who were included. It is not known whether these findings would apply to other or more heterogeneous populations including other races and ethnicities. Although the analysis excluding Asian subjects partly addresses this issue, the problem of population stratification is another limitation of the study. Finally, although three primary outcome measures were selected a priori, no additional correction was made for multiple comparisons or multiple phenotypes apart from the use of ANCOVA.
In summary, this study showed that amphetamine produced smaller effects in individuals with a particular DAT1 genotype. The results are highly consistent with recent findings with a different stimulant, methylphenidate, in a different subject population, ADHD patients, and using different outcome measures, clinical outcome. This convergence of findings across different experimental conditions provides a strong case for an important role for the DAT1 genotype in stimulant drug response. More generally, studies that clarify the genetic determinants of drug response will improve our understanding of the factors underlying complex drug effects such as addiction and therapeutic response, and may ultimately allow us to predict an individual's response.

\section{ACKNOWLEDGEMENTS}

This work was supported by NIH (DA02812; RR00055) and by the Jean Young and Walden $\mathrm{W}$ Shaw Foundation. We thank Jen McDonald and Michelle Dassenger for technical assistance.

\section{REFERENCES}

American Psychiatric Association (1994). Diagnostic and Statistical Manual of Mental Disorders, 4th edn. American Psychiatric Press Inc.: Washington, DC.

Brauer LH, de Wit H (1996). Subjective responses to D-amphetamine alone and after pimozide pretreatment in normal, healthy volunteers. Biol Psychiatry 39: 26-32.

Cesarec Z, Nyman AK (1985). Differential response to amphetamine in schizophrenia. Acta Psychiatr Scand 71: 523-538.

Derogatis L (1983). SCL-90-R Manual II. Clinical Psychometric Research: Towson, MD.

de Wit H, Uhlenhuth EH, Johanson CE (1986). Individual differences in the reinforcing and subjective effects of amphetamine and diazepam. Drug Alcohol Depend 16: 341-360.

Fergusson DM, Horwood LJ, Lynskey MT, Madden PAF (2003). Early reactions to cannabis predict later dependence. Arch Gen Psychiatry 60: 1033-1039.

Foltin RW, Fischman MW (1991). Assessment of abuse liability of stimulant drugs in humans: a methodological survey. Drug Alcohol Depend 28: 3-48.

Fuke S, Suo S, Takahashi N, Koike H, Sasagawa N, Ishiura S (2001). The VNTR polymorphism of the human dopamine transporter (DAT1) gene affects gene expression. Pharmacogenom $J$ : $152-156$.

Gabbay FH (2003). Variations in affect following amphetamine and placebo: markers of stimulant drug preference. Exp Clin Psychopharmacol 11: 91-101.

Gelernter J, Kranzler HR, Satel SL, Rao PA (1994). Genetic association between dopamine transporter protein alleles and cocaine-induced paranoia. Neuropsychopharmacology 11: 195-200.

Haertzen CA, Kocher TR, Miyasato K (1983). Reinforcements from the first drug experience can predict later drug habits and/or addiction: results with coffee, cigarettes, alcohol, barbiturates, minor and major tranquilizers, stimulants, marijuana, hallucinogens, heroin, opiates and cocaine. Drug Alcohol Depend 11: 147-165.

Heinz A, Goldman D, Jones DW, Palmour R, Hommer D, Gorey JG et al (2000). Genotype influences in vivo dopamine transporter availability in human striatum. Neuropsychopharmacology 22: 133-139. 
Hutchison KE, Wood MD, Swift R (1999). Personality factors moderate subjective and psychophysiological responses to D-amphetamine in humans. Exp Clin Psychopharmacol 7: 493-501.

Jacobsen LK, Staley JK, Zoghbi SS, Seibyl JP, Kosten TR, Innis RB et al (2000). Prediction of dopamine transporter binding availability by genotype: a preliminary report. Am J Psychiatry 157: 1700-1703.

Johanson CE, Uhlenhuth EH (1980). Drug preference and mood in humans: diazepam. Psychopharmacology (Berl) 71: 269-273.

Jones SR, Gainetdinov RR, Wightman RM, Caron MG (1998). Mechanisms of amphetamine action revealed in mice lacking the dopamine transporter. J Neurosci 18: 1979-1986.

Kang AM, Palmatier MA, Kidd KK (1999). Global variation of a 40bp VNTR in the $3^{\prime}$-untranslated region of the dopamine transporter gene (SLC6A3). Biol Psychiatry 46: 151-160.

Kirley A, Lowe N, Hawi Z, Mullins C, Daly G, Waldman I et al (2003). Association of the $480 \mathrm{bp}$ DAT1 allele with methylphenidate response in a sample of Irish children with ADHD. Am J Med Genet 121B: 50-54.

Loo SK, Specter E, Smolen A, Hopfer C, Teale PD, Reite ML (2003). Functional effects of the DAT1 polymorphism on EEG measures in ADHD. J Am Acad Child Adolesc Psychiatry 42: 986-993.

Martinez D, Gelernter J, Abi-Dargham A, van Dyck CH, Kegeles L, Innis RB et al (2001). The variable number of tandem repeats polymorphism of the dopamine transporter gene is not associated with significant change in dopamine transporter phenotype in humans. Neuropsychopharmacology 24: 553-560.

Martin WR, Sloan JW, Sapira JD, Jasinski DR (1971). Physiologic, subjective, and behavioral effects of amphetamine, methamphetamine, ephedrine, phenmetrazine, and methylphenidate in man. Clin Pharmacol Ther 12: 245-258.

Mattay VS, Goldberg TE, Fera F, Hariri AR, Tessitore A, Egan MF et al (2003). Catechol $O$-methyltransferase val158-met genotype and individual variation in the brain response to amphetamine. Proc Natl Acad Sci USA 100: 6186-6191.

McNair D, Lorr M, Droppleman DL (1971). Profile of Mood States. Educational and Industrial Testing Service: San Diego.

Mill J, Asherson P, Browes C, D'Souza U, Craig I (2002). Expression of the dopamine transporter gene is regulated by the $3^{\prime}$ UTR VNTR: evidence from brain and lymphocytes using quantitative RT-PCR. Am J Med Genet 114: 975-979.

Mitchell SH, Laurent CL, de Wit H (1996). Interaction of expectancy and the pharmacological effects of D-amphetamine: subjective effects and self-administration. Psychopharmacology (Berl) 125: 371-378.

Mitler MM, Hajdukovic R, Erman MK (1993). Treatment of narcolepsy with methamphetamine. Sleep 16: 306-317.

Roman T, Rohde LA, Hutz MH (2004). Polymorphisms of the dopamine transporter gene: influence on response to methylphenidate in attention deficit-hyperactivity disorder. $\mathrm{Am} \mathrm{J}$ Pharmacogenom 4: 83-92.

Selzer ML (1971). The Michigan Alcoholism Screening Test: the quest for a new diagnostic instrument. Am J Psychiatry 127: 1653-1658.

Spencer T, Biederman J, Wilens T, Harding M, O'Donnell D, Griffin S (1996). Pharmacotherapy of attention-deficit hyperactivity disorder across the life cycle. J Am Acad Child Adolesc Psychiatry 35: 409-432.

Spielewoy C, Biala G, Roubert C, Hamon M, Betancur C, Giros B (2001). Hypolocomotor effects of acute and daily D-amphetamine in mice lacking the dopamine transporter. Psychopharmacology (Berl) 159: 2-9.

Stein MA, Waldman I, Sarampote C, Seymour K, Robb A, Conlon C et al (2002). Dopamine transporter genotype (DAT1) affects stimulant response in children with ADHD. Am J Hum Genet 71: S503.

Uhl GR, Liu QR, Naiman D (2002). Substance abuse vulnerability loci: converging genome scanning data. Trends Genet 18: 420-425.

Vandenbergh DJ, Persico AM, Hawkins AL, Griffin CA, Li X, Jabs EW et al (1992). Human dopamine transporter gene (DAT1) maps to chromosome 5p15.3 and displays a VNTR. Genomics 14: 1104-1106.

White TL, Justice AJ, de Wit H (2002). Differential subjective effects of D-amphetamine by gender, hormone levels and menstrual cycle phase. Pharmacol Biochem Behav 73: 729-741.

Winsberg BG, Comings DE (1999). Association of the dopamine transporter gene (DAT1) with poor methylphenidate response. J Am Acad Child Adolesc Psychiatry 38: 1474-1477. 